\title{
Settling down on chitin
}

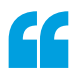

the importance

of pilus-

mediated

interactions

and filament

meshes in

V. cholerae

chitin

colonization
The Gram-negative bacterium

Vibrio cholerae is a facultative

human pathogen responsible for the diarrhoeal disease cholera.

$V$. cholerae has human and environmental reservoirs. In the aquatic environment, $V$. cholerae persists in a planktonic state or in a biofilm on chitinous exoskeletons of crustaceans and zooplankton, which the bacteria utilize as a sole carbon source. Two papers now provide novel insights into how bacteria colonize chitin, revealing the role of a type IV pilus and filamentation.

In the first paper, Blokesch and colleagues investigated $V$. cholerae DNA-uptake pili, which are type IV pili that are produced during growth on chitin and have a role in natural transformation. They showed that DNA-uptake pili are highly dynamic, undergoing extension and retraction before uptake of DNA dependent on

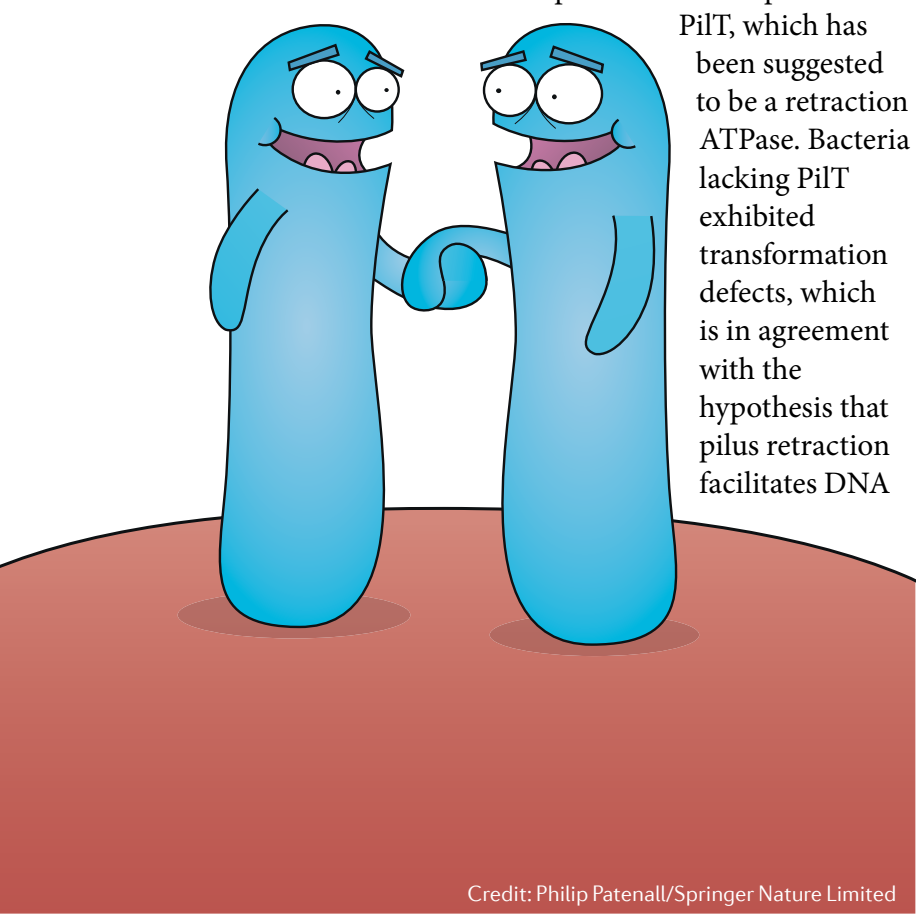

uptake. Interestingly, the authors also noticed that retraction-deficient $\Delta$ pilT cells often aggregated. Complementation of $\Delta p i l T$ cells with PilT restored the transformation defect and abolished the aggregation phenotype, whereas the deletion of components involved in the assembly of the DNA-uptake pilus abolished aggregation. Moreover, they showed that the major subunit of DNA-uptake pili, PilA, mediated direct pilus-pilus interactions. Importantly, the ability to aggregate varied depending on the particular PilA pilin variant, and the authors showed that specific PilA-PilA interactions represent a mechanism for kin recognition. Next, the authors showed that cells colonizing chitin surfaces produced pili that self-interacted and formed a dense mesh-like network across the entire chitin surface, which implies that the chitin surface promotes interactions between pili. In addition, they found that DNA-uptake pili can bind directly to chitin surfaces. This finding, together with the observation that mutants lacking pilA displayed a colonization defect under conditions of continuous mixing, suggests that DNA-uptake pili are required for chitin colonization under flow. On the basis of these results, the authors hypothesize that a major role of DNA-uptake pili in natural environments is to promote inter-bacterial interactions and facilitate chitin colonization.

In a second paper, Nadell and colleagues investigated the role of $V$. cholerae cell morphological variation in chitin colonization. They showed that two pandemic $V$. cholerae serotypes formed filamentous subpopulations under nutrient-limited conditions. Next, the authors compared the ability of filamentous morphotypes and non-filamenting cells to colonize chitin under conditions that mimic the natural marine environment. They found that filamentous $V$. cholerae colonized chitin particles more rapidly than short cells. Moreover, following colonization, biofilm formation by filamentous cells was independent of the matrix polysaccharide VPS and the adhesin RbmA. The authors speculated that the entangled cells themselves provide robustness to the biofilm structure. Having established that filamentation provides an advantage during initial attachment to chitin, the authors carried out longer-term competition experiments and found that non-filamentous biofilms eventually outcompeted and displaced filamentous biofilms, which might be owing to the protective effect that the biofilm matrix provides. However, when the authors periodically introduced new chitin particles that can be colonized, they found that filamentous biofilms remained in the majority against competing non-filamentous cells. Thus, the filamentous morphotype might represent an alternative mode of biofilm architecture that provides an advantage under conditions of rapid chitin particle turnover.

In summary, the findings of the studies uncover the importance of pilus-mediated interactions and filament meshes in $V$. cholerae chitin colonization.

Andrea Du Toit

ORIGINAL ARTICLES Adams, D. W. et al. DNA-uptake pili of Vibrio cholerae are required for chitin colonization and capable of kin recognition via sequence-specific self-interaction. Nat. Microbiol. https://doi.org/10.1038/s41564-019-0479-5 (2019)| Wucher, B. R. et al. Vibrio cholerae filamentation promotes chitin surface attachment at the expense of competition in biofilms. Proc. Natl Acad. Sci.USA https://doi.org/10.1073/pnas.1819016116 (2019) 